\title{
Performance Analysis of a combined WDM/TDM Network based on fixed Wavelength Assignment
}

\author{
J. Späth, J. Charzinski, S. Hörz \\ Institute of Communication Networks and Computer Engineering \\ University of Stuttgart, \\ Pfaffenwaldring 47, D-70569 Stuttgart, Germany, \\ Tel.: +497116857990, e-mail: spaeth@ind.uni-stuttgart.de \\ M. N. Huber \\ Siemens $A G$ \\ Hofmannstr. 51, D-81359 Munich, Germany
}

\begin{abstract}
Photonic telecommunication networks gain increasing importance in various fields extending from access to wide area networks. Due to limitations of fast all-optical switching technology, many promising concepts are based on circuit switching. In this paper, we propose a new and simple star topology using fixed wavelength assignment and a combination of wavelength and time division multiplexing. Owing to the fixed assignment, no tunable components or wavelength converters are needed in the central star element. For this system architecture, we present a performance evaluation based on a probabilistic method to calculate call blocking probabilities, which is validated by simulation and compared to a rough approximation based on a simple loss system. The results allow to evaluate the trade-off between capacity increase either by additional wavelengths or additional time slots in the system.
\end{abstract}

Keywords

Optical Star Network, WDM, TDM, Performance Analysis

\section{INTRODUCTION}

Photonic networks attract raising attention due to several advantages of optical technology like huge transmission capacity of optical fibers and increasing network reliability due to optical transmission. An important aspect is wavelength division multiplexing (WDM), which allows the transportation 
of multiple wavelengths on one fiber without interference. Additionally, this mode profits from EDFAs (Erbium Doped Fiber Amplifiers) which allow the simultaneous amplification of multiple wavelengths.

The increasing interest in photonic networks is manifested by several special issues published lately $[10,11]$. They show that the focus is moving from component oriented research towards network aspects and display the wide scope of applications ranging from on-chip communication to wide area networks (WANs).

The great progress made in the last few years can be seen by a comparison with the state of the art at the beginning of this decade [4]. Numerous network concepts, which can be classified in single-hop and multi-hop systems [17, 18], have been proposed since then. A large part of the proposals considered star based networks which are supposed to be mainly applied as local or metropolitan area networks (LANs, MANs) with higher transmission rates than today [24]. But there were also suggestions to build wide area telecommunication networks using star networks (for example in [8]).

Up to now, most concepts were based on packet transmission mode. Nevertheless, recently circuit switching again attracted more attention [9], since it is possible to show that for some application areas circuit switching offers serious advantages over packet switching [22].

In this work, we will therefore concentrate on the call level in a circuit switched WDM star network. However, instead of a broadcast star coupler as central element, we propose a simple exchange element using fixed wavelength assignment. The network could be favorably applied as a regional network where single end users are connected with moderate bit rates (i.e. a fraction of a wavelength's bandwidth). Most likely, pure WDM systems will play a minor role to connect end users because the bandwidth available on a wavelength cannot be fully utilized by a single user. Moreover, the enhancement with TDM offers a simple way to allow multiple connections for each user. Thus, we will concentrate on combined WDM/TDM systems in this work.

The following section describes the proposed architecture. Section 3 gives an overview on possible performance evaluation methods whereas Section 4 contains the modeling and analysis. After presenting some results we conclude with an outlook on further work.

\section{NETWORK ARCHITECTURE}

For the physical topology a star structure is supposed. However, instead of using a passive star coupler, we apply fixed wavelength routing in the star, which can be realized with simple grating elements [16]. Thus, the wavelength that has to be chosen for a transmission is explicitly determined by the link to which the destination node is connected. Figure 1 shows a simple example with 3 links connected to the star. $m$ end stations (each comprising one transmitter 
and one sender) are connected to every link. They have common access to $a$ time slots carried on each wavelength of a single link.

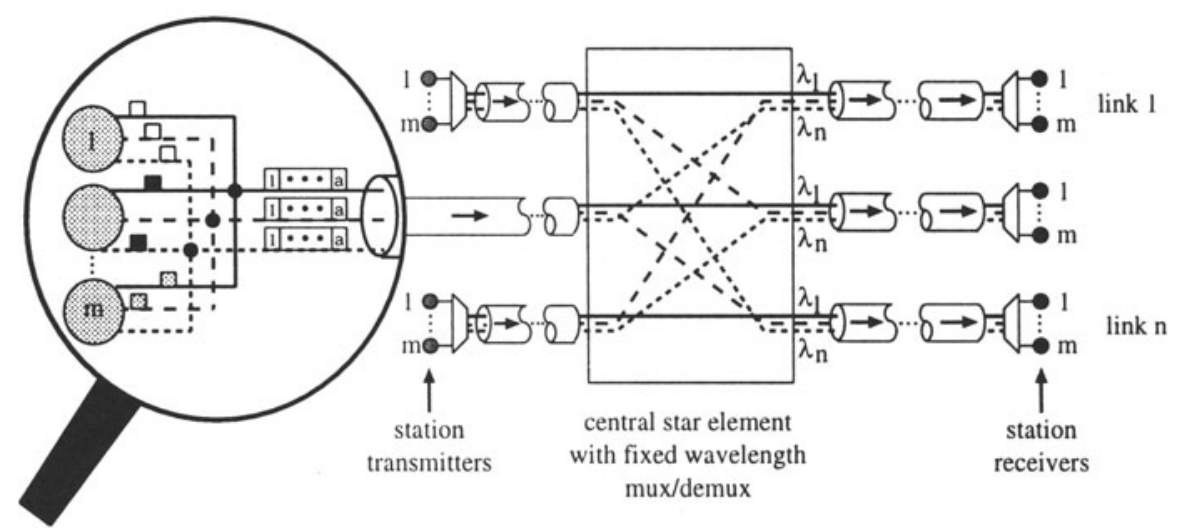

Figure 1 Network architecture

This architecture provides several advantages concerning technological demands and implementation complexity.

- No tunable elements are required in the star exchange element leading to reduced realization cost.

- Fixed wavelength routing offers various advantages. It can be realized using simple and fixed elements. In [8], the principle of a simple grating device is shown allowing wavelength multiplexing and demultiplexing. Progress for integrated wavelength demultiplexers based on waveguide arrays was announced recently for example in [19] and [21]. Moreover, there is no signal splitting amongst multiple links and therefore the power degradation is less than in the case of passive broadcast star couplers. Furthermore, the limited set of available wavelengths can be reused in the proposed fixed arrangement allowing a multiple, simultaneous use of the same wavelength on different links.

- No wavelength converters are required. These elements are still complex and difficult to realize (see for example [25]). In particular to achieve satisfying all-optical solutions, a lot of research is still necessary.

- In our system, we carry multiple time slots of constant length on each wavelength. When a connection is established, the wavelength access is periodically assigned to the stations for a certain period of time. During this time, a transparent channel that is independent of signal format or bit rate is available for the stations. The length of a slot depends on the achievable tuning speed of optical components on the one hand and the maximum allowed packetization delay on the other hand. 
From a technological point of view, several promising results were reported for OTDM (Optical Time Division Multiplexing). A practical use seems realistic in the near future and various groups are working on a quick introduction of OTDM networks [2]. An up to date overview and description of the state of the art is given in [20].

For the described architecture, one wavelength is required for each link connected to the star (i.e. as many wavelengths have to be transported in WDM mode as links are connected). The architecture could be extended by using multiple fibers for each link. Nevertheless, this requires more resources (i.e. transmitter and receiver) at the stations. Moreover, bidirectional transmission on a wavelength by using directed couplers could be used instead of using one fiber for each direction as shown in Figure 1.

To achieve a real system, some extensions would be necessary like for example the implementation of a network control and management instance as well as signaling facilities.

In combined WDM/TDM systems it is of high interest to work out the relations between the parameters like number of WDM channels, bit rate per channel, and number of fibers. Recently, some work was reported to evaluate the trade-off between these parameters from an economical and technological point of view $[7,15]$. Another interesting perspective is a comparison with regard to traffic theoretical aspects. In the following, we present a method which allows a comparison of call blocking probabilities.

\section{PERFORMANCE EVALUATION METHODS}

A number of performance evaluation methods for WDM based star networks are reported in literature. In [14], Lu and Kleinrock consider a packet transmission star network with uniform and non-uniform traffic as loss system, i.e. blocked packets are discarded. A mathematical model is derived based on birth-death processes which lead to Markov chains for resource occupancies (i.e. wavelength, transmitter, and receiver occupancies).

For circuit-switched WDM/TDM networks, many results can be adapted from earlier work on "classical" electrical networks. In [12] an approximation is introduced while [5] presents a decomposition method that allows the exact calculation for call blocking probabilities in circuit-switched networks. The latter method does not depend on the routing matrix and focuses on computational savings for large arbitrarily meshed network structures.

Other work is concerned with call blocking probabilities in circuit-switched integrated services networks. In [6], a loss system is considered with a variable number of channels per call. However, the method assumes fixed routing and has to make several approximations for larger networks.

Recently, Awater et al. presented an exact computation of time and call blocking probabilities in multi-traffic, multi-resource loss systems with com- 
pletely shared resources and gave also an overview of related work [1]. The proposed algorithms were stated to be stable and to achieve an efficiency comparable to earlier proposed approximations.

For performance investigations of systems like the one described in Section 2 , there are several suitable methods:

- Analyzing the state space of a system leads to exact results for pure chance traffic. However, it is necessary that each state of the system and all transition rates between any two states are considered. Therefore, this method results in large systems of linear equations practically unsolvable for networks with realistic complexity. Numerous approximations were deployed in the past to reduce computing requirements.

- Another group of approximations comprises transformations to well known link systems as spatial equivalents. In this field, numerous methods for investigations of multi stage system as for example iterative improvement of supposed link occupancy distributions are reported in literature (see the detailed overview in [13]). But up to our knowledge, there is no method directly adaptable to our problem.

- Probabilistic methods combined with permutation investigations can also lead to exact results. Due to the high complexity of real systems however, simplifying assumptions as for example simple traffic characteristics and statistical independence of states are often necessary.

- In principle, simulative investigations allow an examination of any system. However, there exists also a trade-off between accuracy and computing effort. Nevertheless, simulations are a valuable tool in confirming analytical results. Moreover, they allow extensions to traffic types or protocols which are not analytically tractable.

In the following, we present a probabilistic method to investigate our system. The analytical results are verified by simulation.

\section{MODELING AND ANALYSIS}

Table 1 lists the parameters used for the following analysis.

$n$ number of links (equals number of wavelengths)

$m$ number of stations per link

a number of time slots per wavelength

$\lambda$ rate of connection requests from each station

$\varepsilon \quad$ reciprocal mean holding time of a connection

Table 1 System parameters for analysis 
Each connection between two end users occupies one time slot. A source selects a receiver randomly out of the $n \cdot m$ stations connected to the network* ${ }^{*}$. The optical wavelength which the new connection will be carried on is determined by the fixed wavelength routing scheme introduced before. Let $S$ be the random variable denoting the number of time slots occupied at the source transmitter just before the new connection is to be set up. Let $J$ be the random variable for the occupation of the sending channel, i.e. the number of time slots occupied on the wavelength which must be used to route the connection to the selected receiver. Finally, let $D$ be the random variable for the number of time slots occupied at the destination station's receiver.

Assuming stationarity, we have the following Erlang probability distributions for loss systems for $S, J$ and $D$. Here, the loss due to blocking at other places is neglected.

$$
\begin{aligned}
& \mathrm{P}\{S=s\}=\frac{\frac{A_{S}^{s}}{s !}}{\sum_{i=0}^{a} \frac{A_{S}^{i}}{i !}} \quad \text { for } 0 \leq s \leq a \\
& \mathrm{P}\{D=d\}=\frac{\frac{A_{S}^{d}}{d !}}{\sum_{i=0}^{a} \frac{A_{S}^{i}}{i !}} \quad \text { for } 0 \leq d \leq a \\
& \mathrm{P}\{J=j\}=\frac{\frac{A_{W}^{j}}{j !}}{\sum_{i=0}^{a} \frac{A_{W}^{i}}{i !}} \quad \text { for } 0 \leq j \leq a
\end{aligned}
$$

In equations (1) and (2), the offered traffic per station $A_{S}$ is given by

$$
A_{S}=\frac{\lambda}{\varepsilon}
$$

and in equation (3) the offered traffic per wavelength $A_{W}$ is given by

$$
A_{W}=\frac{m}{n} \cdot A_{S}
$$

Assuming independence** of $S$ and $J$ and random selection of one out of the free time slots at connection set-up, we find the distribution for the number of

\footnotetext{
* The case of a source connecting to itself is not excluded from the following analysis because it would make the analysis much more complex without significantly changing the results. **This assumption is quite accurate owing to the fact that $m$ users have access to each wavelength and the connections of a single user are uniformly distributed over all $n$ wavelengths on a link.
} 
time slots $Q$ which are free at the source transmitter and on the wavelength:

$$
\begin{aligned}
\mathrm{P}\{Q=q \mid S=s, J=j\}= & \frac{\left(\begin{array}{c}
a-s \\
q
\end{array}\right)\left(\begin{array}{c}
s \\
a-j-q
\end{array}\right)}{\left(\begin{array}{c}
a \\
a-j
\end{array}\right)} \\
& \text { for } q \in[\max \{0, a-j-s\}, \min \{a-j, a-s\}]
\end{aligned}
$$

Now, proceeding to the destination receiver, we are looking for the probability of loss under the condition that we can choose from $Q$ time slots on the wavelength and there are $D$ busy time slots at the receiver. Loss occurs if all $Q$ time slots are busy at the receiver:

$$
\begin{aligned}
\mathrm{P}\{\operatorname{loss} \mid Q=q, D=d\}= & \frac{\left(\begin{array}{l}
d \\
q
\end{array}\right)}{\left(\begin{array}{l}
a \\
q
\end{array}\right)} \\
& \text { for } q \leq d
\end{aligned}
$$

Defining the binomial coefficient $\left(\begin{array}{l}a \\ b\end{array}\right)$ to be zero for $a<b, a<0$ or $b<0$ and taking $S, J$ and $D$ to be independent, we obtain the loss probability from (6) and (7):

$$
\begin{aligned}
B= & \sum_{s=0}^{a} \sum_{j=0}^{a} \sum_{d=0}^{a} \sum_{q=0}^{a} \mathrm{P}\{\operatorname{loss} \mid Q=q, D=d\} \cdot \mathrm{P}\{Q=q \mid S=s, J=j\} \\
& \cdot \mathrm{P}\{S=s\} \mathrm{P}\{J=j\} \mathrm{P}\{D=d\} \\
= & \sum_{s=0}^{a} \sum_{j=0}^{a} \sum_{d=0}^{a} \sum_{q=0}^{a} \mathrm{P}\{S=s\} \mathrm{P}\{J=j\} \mathrm{P}\{D=d\} \frac{\left(\begin{array}{c}
a-s \\
q
\end{array}\right)\left(\begin{array}{c}
s \\
a-j-q
\end{array}\right)}{\left(\begin{array}{c}
a \\
a-j
\end{array}\right)} \frac{\left(\begin{array}{l}
d \\
q
\end{array}\right)}{\left(\begin{array}{l}
a \\
q
\end{array}\right)}
\end{aligned}
$$

\section{RESULTS}

Comparison for systems with a given number of stations

Using the parameters of our system according to table 1, we get the total number of stations in the system $m_{\text {total }}$ :

$m_{\text {total }}=n \cdot m$

Moreover, the number of resources (i.e. slots) per link is given by

$R_{l i n k}=n \cdot a$

and the number of resources in the star element by

$R_{\text {star }}=n^{2} \cdot a$ 


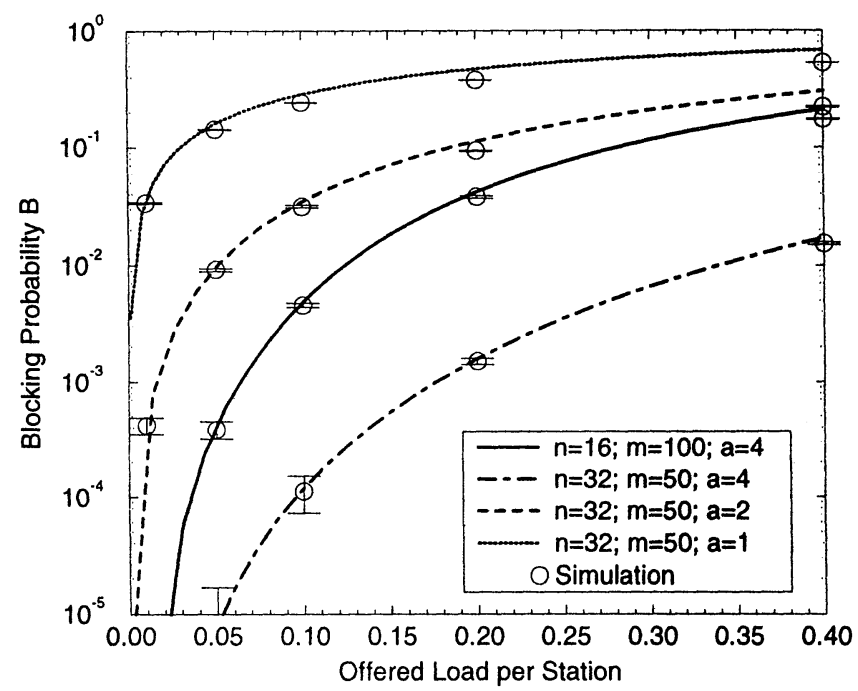

Figure 2 Call blocking probability for $m_{\text {total }}=m \cdot n=1600$ stations

Figure 2 shows the call blocking probability versus the offered load per station $A_{S}$ for a system with $m_{\text {total }}=1600$ stations. The solid line represents a system where $m=100$ stations are connected to each of the $n=16$ links* and $a=4$ time slots are available on each of the 16 required wavelengths (we name this system reference system in the following).

The dashed lines describe also a network with $m_{\text {total }}=1600$ stations, but now using $n=32$ links with $m=50$ stations per link. It can be seen that for the same value of $a=4$ the blocking probability decreases significantly due to the reduced traffic per wavelength. Compared to the reference system, $a=2$ leads to higher blocking probabilities although $R_{\text {link }}$ is constant and $R_{\text {star }}$ is even doubled. This is due to the smaller bundles resulting in a smaller economy of scale. For the case $a=1$, leading to the same $R_{\text {star }}$ as in the reference system, blocking probabilities are even higher.

Figure 2 also contains simulation results which are depicted with a 0.95 confidence interval in all figures. They indicate that the analytical results are overestimating the exact values for high loads. This effect is due to the influence of the correlation between the link, transmitter and receiver states which we neglected in our analysis. This correlation slightly decreases the blocking probabilities at high traffic load per station.

* At present, a number of channels in a WDM system in the range of 8 to 32 is under discussion at standardization bodies. 


\section{System Scalability}

Figure 3 shows the influence of system scaling on blocking probabilities. Compared to our reference system, increasing $m$ to $m=200$ and therefore doubling $m_{\text {total }}$ leads to higher blocking probabilities as expected. However, if we simultaneously double the number of time slots $a$ to $a=8$ thus holding $R_{\text {link }} / m$ constant, we see lower blocking probabilities. This can be explained by the economy of scale.

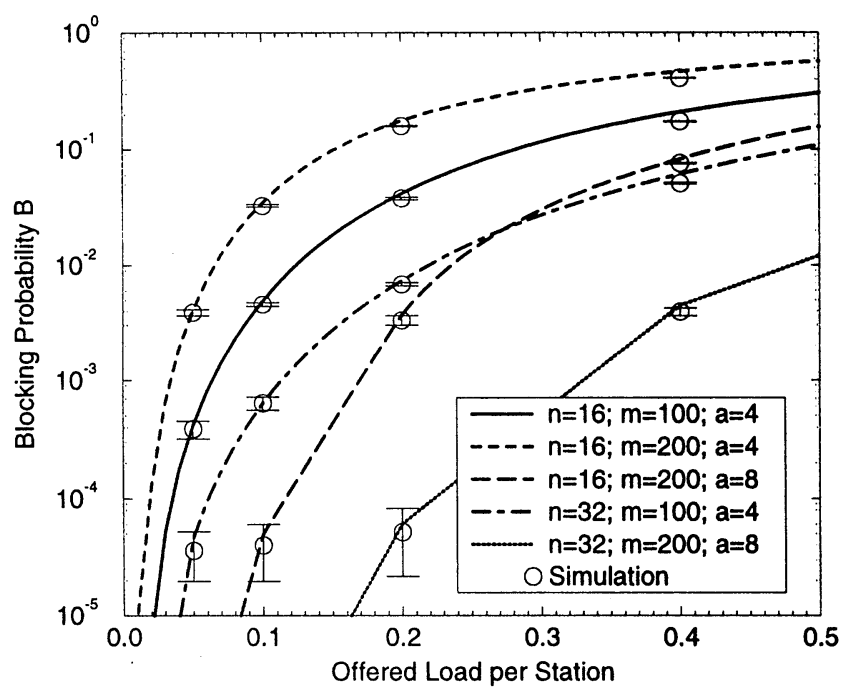

Figure 3 Blocking probability for scaled systems

Another way of doubling the system size is doubling the number of attached links $(n=32)$. If $m$ and $a$ are not changed, we see a reduction of blocking probabilities although $m_{\text {total }}$ is doubled. This can be explained by the increase of resources according to (10) and (11). As $R_{\text {link }}$ grows proportional to $n$, the number of resources available for a bundle of $m$ stations is doubled. Moreover, $R_{s t a r}$ grows quadratically with $n$.

This is also the reason that the results for twice as many stations per link ( $n=32, m=200, a=4)$ are identical to the reference system although now $m_{\text {total }}$ equals 6400 . Obviously, blocking probabilities decrease further if we additionally raise $a$ to $a=8$.

For high traffic load per station, we see again slightly higher blocking in the analytical results compared to simulation, as discussed before.

It can be seen that system scaling based on additional links improves the performance while the number of time slots per wavelength (and thus the re- 
quired tuning speed for each station) remains the same. However, the effect of decreasing blocking probabilities with increasing number of stations is limited by the number of wavelengths which have to be transported in WDM mode. Moreover, the stations are more complex if they have to support a higher number of wavelengths.

\section{Approximation}

Finally, we present a simple approximation for our system based on a $M / M / a$ loss system. Here, we neglect sender and receiver blocking and approximate our system with a loss system for each wavelength on a link. The number of servers equals a (i.e. the number of time slots on a wavelength) and the offered load is $A_{W}$ as given by equation (5).

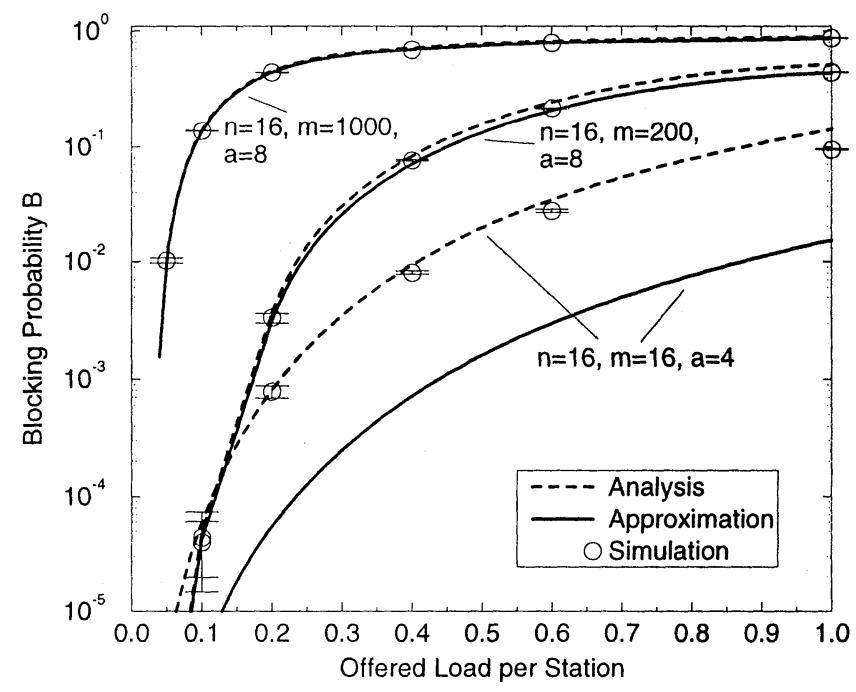

Figure 4 Comparison of exact and approximate solution

Figure 4 shows the blocking probabilities calculated according to (8) (dashed lines) and the results of the approximation (solid lines) versus the offered load per station $A_{S}$. It can be seen that for $m>n$ the approximation is very accurate. However, with decreasing the ratio $m / n$, the approximated blocking probabilities are too low because sender and receiver collisions are neglected. Moreover, the simulation corresponds with the exact analysis for a wide range of $A_{S}$. The approximate results can be improved by additionally considering sender and receiver collisions with simple loss systems. Nevertheless, this leads 
only to a small improvement and the results remain below the simulated values. Thus, formula (8) is especially useful for $m / n$ not too large.

\section{CONCLUSION}

In this paper, we proposed a new WDM/TDM network architecture based on a simple star exchange using fixed wavelength assignment. For this architecture, we presented a performance evaluation based on a probabilistic method to calculate call blocking probabilities for circuit switching mode. Moreover, we depicted a simple approximation which leads to quite accurate results for specific parameter choices. The analytical results allow the evaluation of the trade-off between wavelength and time slot resources in the network.

The proposed model can be extended in several ways. An interesting field already well known in classical networks [3] is the influence of hunting modes for the available time slots. Instead of implementing a random selection of free time slots, the performance could be increased by other searching modes like for example first free first. Moreover, time slot interchanging or wavelength routing in the star element or even time slot rearrangement for existing connections [23] could be considered. However, these extensions will lead to increasing system complexity as well as analytical complexity.

\section{Acknowledgement}

Parts of this work have been supported by the German BMBF (Bundesministerium für Bildung, Wissenschaft, Forschung und Technologie) under contract 01 BP $504 / 2$.

\section{REFERENCES}

[1] G.A. Awater, H.A.B. VAN DE VlaG, "Exact computation of time and call blocking probabilities in large, multi-traffic, multi-resource loss systems", Performance Evaluation, Vol. 25, no. 1, pp. 41-58, March 1996

[2] R.A. Barry, V.W.S. Chan, K.L.Hall et al., "All-Optical Network Consortium - Ultrafast TDM Networks", IEEE Journal on Selected Areas in Communications, Vol. 14, no. 5, pp. 999-1013, June 1996

[3] D. Bazlen, G. Kampe, A. Lotze, "On the Influence of Hunting Mode and Link Wiring on the Loss of Link Systems", Proceedings of the 7th International Teletraffic Congress, pp. 232/1-232/12, Stockholm, 1973

[4] C.A. Brackett, "Dense Wavelength Division Multiplexing Networks: Principles and Applications", IEEE Journal on Selected Areas in Communications, Vol. 8, no. 6, pp. 948-964, August 1990

[5] A.E. Conway, E. Pinsky, "A Decomposition Method for the Exact Analysis of Circuit-Switched Networks", Proceedings of IEEE Infocom 
'92, pp. 996-1003

[6] Z. Dziong, J.W. Roberts, "Congestion Probabilities in a CircuitSwitched Integrated Services Network", Performance Evaluation, Vol. 7 (1987), pp. 267-284

[7] L. Gillner, M. Gustavsson, "Scalability of Optical Multiwavelength Switching Networks: Power Budget Analysis", IEEE Journal on Selected Areas in Communications, Vol. 14, no. 5, pp. 952-961, June 1996

[8] I. Hawker, V. Tandon, D. Cotter, A. Hill, "New Network Infrastructures for the 21st Century, Part 1", British Telecommunications Engineering, July 1994, pp. 103-111

[9] Special Issue on Circuit Switching for Broadband ISDN and Beyond, IEEE Journal on Selected Areas in Communications, Vol. 14, no.2, February 1996

[10] Special Issue on Optical Networks, IEEE Journal on Selected Areas in Communications, Vol. 14, no.5, June 1996

[11] Special Issue on Multiwavelength Optical Technology and Networks, IEEE/OSA Journal of Lightwave Technology, Vol. 14, no. 6, June 1996

[12] F.P. Kelly, "Blocking Probabilities in Large Circuit-Switched Networks", Advances in Applied Probability, Vol. 18 (1986), pp. 473-505

[13] K. KÜMMERLE, Berechnungsverfahren für mehrstufige Koppelanordnungen mit konjugierter Durchschaltung, Institute of Communications Switching and Data Technics, University of Stuttgart, 1969

[14] J.C. LU, L. KLEINRocK, "Performance Analysis of Single-Hop Wavelength Division Multiple Access Networks", Journal of High Speed Networks, Vol. 1 (1992), no. 1, pp. 61-77

[15] G. Marone, "Comparison between WDM and TDM techniques", CSELT Technical Reports, Vol. XXIV, no. 3, pp. 535-548, June 1996

[16] D.J.G. Mestdagh, Fundamentals of Multiaccess Optical Fiber Networks, Artech House, 1995

[17] B. MukherJee, "WDM-Based Local Lightwave Networks; Part I: Single-Hop Systems", IEEE Network, Vol. 6, no. 3, pp. 12-27, May 1992

[18] B. MukherJee, "WDM-Based Local Lightwave Networks; Part II: Multihop Systems", IEEE Network, Vol. 6, no. 4, pp. 20-32, July 1992

[19] H. Okayama, M. Kawahara, T. Kamijoh, "Reflective Waveguide Array Demultiplexer in LiNbO3", IEEE/OSA Journal of Lightwave Technology, Vol. 14, no. 6, pp. 985-990, June 1996

[20] S.-W. Seo, K. Bergman, P.R. Prucnal, "Transparent Optical Networks with Time-Division Multiplexing", IEEE Journal on Selected Areas in Communications, Vol. 14, no. 5, pp. 1039-1051, June 1996

[21] L.H. Spiekman, M.R. Amersfoort, A.H. de Vreede et al., "Design and Realization of Polarization Independent Phased Array Wavelength Demultiplexers using Different Array Orders for TE and TM", 
IEEE/OSA Journal of Lightwave Technology, Vol. 14, no. 6, pp. 991995, June 1996

[22] R.A. Thompson, "Operational Domains for Circuit- and PacketSwitching", IEEE Journal on Selected Areas in Communications, Vol. 14, no. 2, pp. 293-297, February 1996

[23] T. Tomioka, S. Ohshima, "Connection-Based Optical Wavelength Division Multiplexed Network with Time Slot Rearrangement", IEICE Transactions on Communications, Vol. E78-B, no. 9, pp. 1295-1300, September 1995

[24] H.R. van As, "Media access techniques: The evolution towards terabit/s LANs and MANs", Computer Networks and ISDN Systems, no. 26, 1994, pp. 603-656

[25] S.J.B. Yoo, "Wavelength Conversion Technologies for WDM Network Applications", IEEE/OSA Journal of Lightwave Technology, Vol. 14, no. 6, pp. 955-966, June 1996 